\title{
Selective Inhibition of Initiator versus Executioner Caspases Using Small Peptides Containing Unnatural Amino Acids
}

\author{
Chris J. Vickers, ${ }^{\dagger}$ Gonzalo E. González-Páez, ${ }^{\dagger}$ Kevin M. Litwin, ${ }^{\dagger}$ Jeffrey C. Umotoy, ${ }^{\dagger}$ \\ Evangelos A. Coutsias, ${ }^{\ddagger}$ and Dennis W. Wolan* ${ }^{\dagger}$ \\ ${ }^{\dagger}$ Departments of Molecular and Experimental Medicine and Chemical Physiology, The Scripps Research Institute, La Jolla, California \\ 92037, United States \\ "Department of Applied Mathematics and Statistics and Laufer Center for Physical and Quantitative Biology, Stony Brook University, \\ Stony Brook, New York 11794, United States
}

\section{Supporting Information}

ABSTRACT: Caspases are fundamental to many essential biological processes, including apoptosis, differentiation, and inflammation. Unregulated caspase activity is also implicated in the development and progression of several diseases, such as cancer, neurodegenerative disorders, and sepsis. Unfortunately, it is difficult to determine exactly which caspase(s) of the 11 isoforms that humans express is responsible for specific biological functions. This lack of resolution is primarily due to highly homologous active sites and overlapping substrates. Currently available peptide-based inhibitors and probes are based on specificity garnered from peptide substrate libraries. For example, the canonical

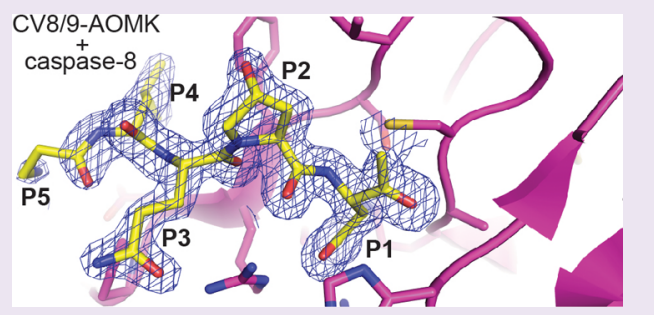
tetrapeptide LETD was discovered as the canonical sequence that is optimally recognized by caspase-8; however, LETD-based inhibitors and substrates promiscuously bind to other isoforms with equal affinity, including caspases-3, -6 , and -9 . In order to mitigate this problem, we report the identification of a new series of compounds that are $>100$-fold selective for inhibiting the initiator caspases- 8 and -9 over the executioner caspases-3, -6 , and -7 .

$\circlearrowleft$ asp aspases are a family of cysteine-dependent aspartatedirected proteases with 11 human isoforms that are traditionally known for their indispensible roles in the initiation (caspases-2, -8, -9, -10) and execution (caspases-3, -6, -7) of apoptosis. ${ }^{1}$ Other family members, including caspases- $1,-4$, and -5 , are important regulators of inflammation and induce pyroptotic cell death as a result of microbial infection. ${ }^{2}$ Intriguingly, recent reports implicate caspase activity as being critical for a variety of other essential biological processes, such as DNA repair signaling and tumor suppression, ${ }^{3}$ skeletal muscle differentiation, ${ }^{4} \mathrm{~B}$-cell proliferation, ${ }^{5}$ dendritic pruning and neuronal plasticity, ${ }^{6}$ embryonic stem cell differentiation through Nanog cleavage, ${ }^{7}$ nuclear factor kappa-light-chainenhancer of activated B cells (NF- $\kappa \mathrm{B})$ activation, lymphocyte and monocyte differentiation and development, ${ }^{9}$ and keratinocyte differentiation and skin barrier formation. ${ }^{10}$ The utility of caspase-dependent proteolysis in an array of cellular functions is continually expanding, and additional caspase roles will likely be discovered with the development of highly potent and specific chemical inhibitors and probes.

Active caspases have many significant irreversible consequences and as such are stored as inactive proenzymes, or procaspases, inside of the cell. ${ }^{11}$ Intriguingly, several small molecules have been identified in high-throughput screens that promote executioner procaspase maturation using in vitro and in vivo models. ${ }^{12-14}$

Aberrant caspase activity is implicated in the development and progression of several diseases, such as neurodegenerative disorders, ${ }^{15}$ cancer $^{16}$ cardiovascular disease, ${ }^{17}$ and sepsis. ${ }^{18}$ Moreover, caspases represent potential drug discovery targets for the treatment of these diseases, and it is therefore imperative to elucidate the exact biological roles of each individual caspase isoform within cell-based assays and animal models. The development of highly selective chemical inhibitors would enable the interrogation of specific caspases, and such compounds are more versatile than standard biological techniques, such as gene knockout, ${ }^{19} \mathrm{~N}$-terminal labeling and mass spectrometry, ${ }^{20}$ and genetically engineering cell lines that express mutant caspases. ${ }^{21}$ Recently, we described activity-based probes and inhibitors for caspase- 3 that have $>100$-fold selectivity over the highly homologous caspase- $7 .^{22,23}$ Another report details an inhibitor (M867) with 64-fold selectivity for caspase- 3 over caspase- $7 .^{24}$ Herein, we describe the discovery and characterization of CV8/9-AOMK and CV8/ 9-KE, which have nanomolar potency and $>100$-fold selectivity for inhibition of initiator caspases- 8 and -9 over executioner caspases-3, -6, and -7 (Supplementary Figure S1).

Commercially available peptide-based inhibitors of caspases typically contain customized tetrapeptide recognition units, which were largely determined by positional-scanning combinatorial libraries (PSCLs) using natural amino acids. ${ }^{25}$ These molecules have been shown to be reasonably nonselective

Received: $\quad$ May 28, 2014

Accepted: July 31, 2014 

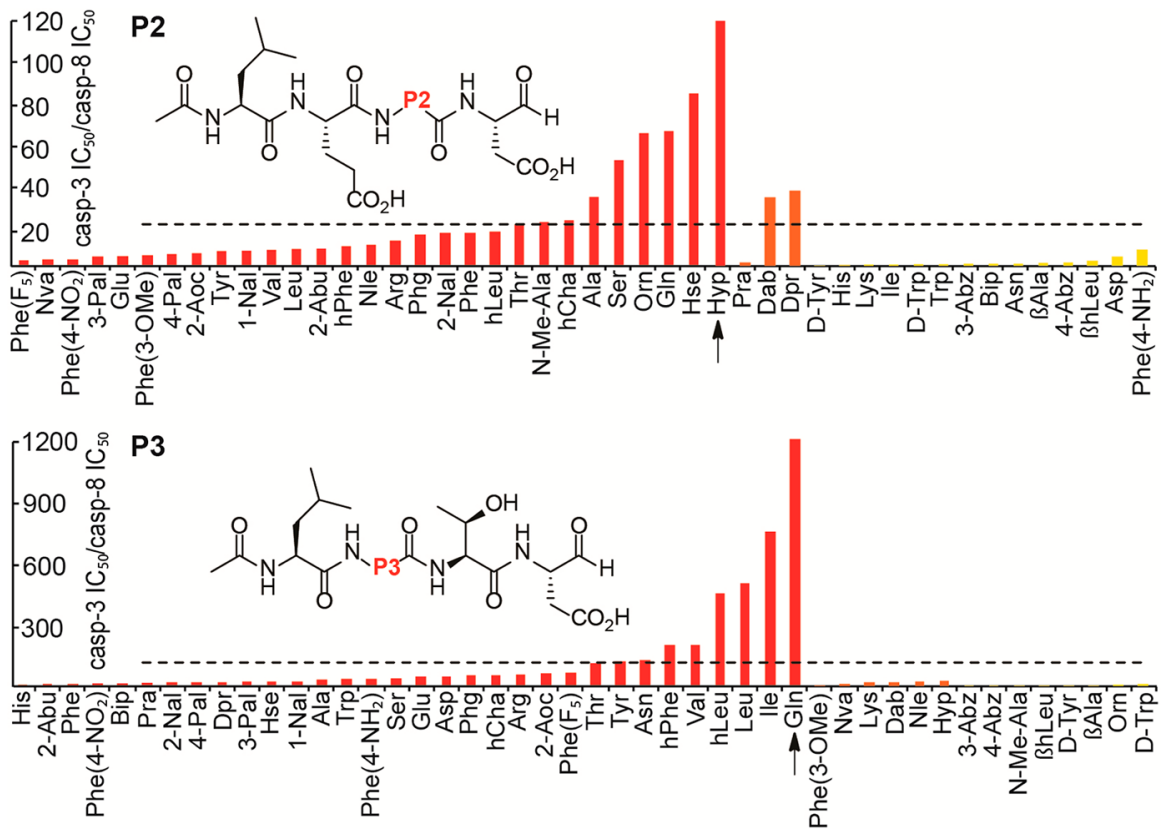

Figure 1. Selectivity screen for caspase-8 inhibitors using single amino acid substitutions at P2 and P3 into the canonical LETD tetrapeptide. Residues that imparted the most caspase- 8 selectivity in comparison to caspase- 3 are labeled with an arrow and were used in subsequent inhibitor design. Inhibitors with high, medium, or low potency are colored red, orange, or yellow, respectively. The horizontal dotted line highlights the level of relative selectivity that is obtained by the canonical caspase- 8 inhibitor Ac-LETD-CHO.

A

HO P2

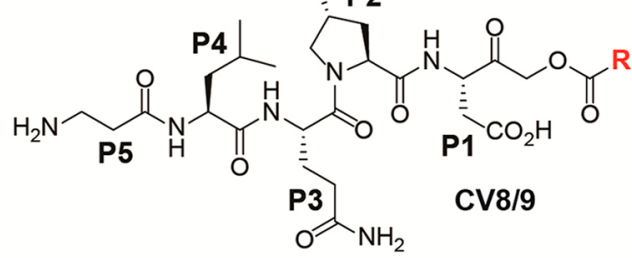<smiles>Cc1cccc(-c2cccc(C)c2C(=O)O)c1NC(=O)O</smiles>

B

\begin{tabular}{c|ccccc} 
& \multicolumn{5}{c}{$\mathrm{IC}_{50}$ values $(\mu \mathrm{M})$} \\
& casp-3 & casp-6 & casp-7 & casp-8 & casp-9 \\
\hline Ac-LETD-AOMK & 0.020 & 0.068 & 0.46 & 0.028 & 0.089 \\
CV8/9-AOMK & 43 & 220 & 250 & 0.30 & 0.32 \\
CV8/9-KE & 150 & 320 & 400 & 0.30 & 0.64
\end{tabular}

Figure 2. (A) Chemical structure of CV8/9 series compounds that are optimized for selective inhibition of initiator caspases-8 and -9 over executioner caspases-3, -6, and -7. (B) $\mathrm{IC}_{50}$ data for LETD and CV8/9-based inhibitors against a panel of recombinant caspases. Each inhibitor/ caspase pair was preincubated at RT for $1 \mathrm{~min}$, followed by the addition of caspase substrate to a final concentration of $50 \mu \mathrm{M}$ and immediate determination of the initial reaction velocity $(\mathrm{RFU} / \mathrm{s})$.

across the caspase family, especially among executioner and initiator caspases. ${ }^{26}$ On the basis of our previous success finding caspase-3-selective compounds, we synthesized individual peptide libraries incorporating chemically diverse unnatural amino acids in a search for specific inhibitors of caspase- 8 over caspase-3 (Figure 1). ${ }^{22,27}$

The canonical tetrapeptide recognition sequence for caspase8 is LETD (labeled P4-P1 from N- to C-terminus), and we preserved the obligatory P1 aspartic acid residue within the peptide library. Positions P2 through P4 of the LETD sequence were each substituted with 46 different natural and unnatural amino acids to identify inhibitors with higher caspase- 3 versus caspase-8 $\mathrm{IC}_{50}$ ratios (Figure 1). Each inhibitor included a Cterminal aldehyde warhead, which is desirable for two reasons: (1) aldehyde warheads can be derived from solid-support, simplifying the synthesis of each compound, and (2) reversible aldehyde warheads are less potent than their irreversible counterparts and allow more for a more direct comparison of selective residues among the two proteases. To this point, caspase isoform selectivity decreases approximately 10 -fold when transitioning from a reversible aldehyde to an irreversible acyloxymethyl ketone (AOMK) warhead that is commonly employed for caspase inhibitors and probes (Figures 1 and 2, data not shown).

The library of P2 permutations identified several residues that provided enhanced selectivity over the canonical threonine, including hydroxyproline (Hyp) and homoserine (Hse) that had 125- and 83-fold selectivity, respectively (Figure 1). Strikingly, upon synthesis of a diverse pool of P3 derivatives, several substitutions imparted $>100$-fold selectivity for inhibiting caspase-8 over caspase-3, such as glutamine (Gln) and isoleucine (Ile), which had 1200- and 750-fold selectivity, respectively (Figure 1). Substitution of the P4 residue with 46 chemically distinct substitutions did not provide a residue that 
was more selective than the canonical leucine (Leu) (Supplementary Figure S2). Similarly, appending different residues at P5 did not yield significant additional selectivity; however, $\beta$-alanine $(\beta \mathrm{Ala})$ was chosen as a $\mathrm{N}$-terminal modification to increase water solubility (Supplementary Figure S2).

After testing 184 compounds for their ability to selectively inhibit caspase- 8 compared to caspase-3, we identified hydroxyproline at $\mathrm{P} 2$, glutamine at $\mathrm{P} 3$, leucine at $\mathrm{P} 4$, and $\beta$ alanine at P5 that when combined yielded a highly selective caspase-8 inhibitor (Figure 2). This new sequence was compared to the inhibitory properties of Ac-LETD-AOMK, which is commonly used to target caspase-8 (Figure 2). We tested Ac-LETD-AOMK in vitro against initiator caspases- 8 and -9 , as well as the executioner caspases- $3,-6$, and -7 , and found that the LETD-based compound was highly promiscuous across this series of caspases with the exception of slight selectivity against caspase-7 (Figure 2).

We next synthesized our new peptide recognition sequence $\mathrm{H}_{2} \mathrm{~N}$ - $\beta$ Ala-L-Q-Hyp-D (termed CV8/9) with an AOMK warhead and found that this compound had nanomolar potency against recombinant initiator caspases- 8 and -9 (Figure 2). Importantly, CV8/9-AOMK had significant selectivity over the executioner caspases-3 (140-fold), -6 (730-fold), and -7 (830-fold) (Figure 2). From our previous studies characterizing different warhead leaving groups, we identified several new functionalities that increase specificity for caspase- 8 inhibition versus caspase-3. One such leaving group that was assessed for improved initiator caspase selectivity was a thiophene moiety, termed KE (ketoester), which was substituted for AOMK into the CV8/9 peptide sequence (Figure 2). ${ }^{23} \mathrm{CV} 8 / 9-\mathrm{KE}$ retained the same $\mathrm{IC}_{50}$ against caspase- 8 but significantly reduced the ability to inhibit the executioner caspases-3 (500-fold), -6 (1000-fold), and -7 (1300-fold) (Figure 2). Notably, this change caused potency against caspase-9 to drop 2-fold (Figure 2).

We further characterized CV8/9-AOMK and Ac-LETDAOMK using irreversible kinetics. ${ }^{28}$ Here, $K_{i}$ and $k_{\text {inact }}$ values can be obtained to describe the initial reversible interaction and subsequent irreversible covalent binding, respectively, of the inhibitor to each caspase (Figure 3). This is accomplished by

\begin{tabular}{|c|c|c|c|}
\hline \multirow[t]{2}{*}{$E+$} & $+1 \frac{K_{i}}{\text { reversit }}$ & $\overrightarrow{\mathrm{ble}} \mathrm{E}-\mathrm{I}$ ir & $\underset{\text { rreversible }}{\stackrel{k_{\text {inact }}}{\longrightarrow}} \mathrm{El}$ \\
\hline & $k_{\text {inact }}\left(\mathrm{s}^{-1}\right)$ & $\begin{array}{l}\text { CV8/9-A } \\
K_{i}(\mathrm{nM})\end{array}$ & $\underset{k_{\text {inact }} / K_{i}\left(\mathrm{mM}^{-1} \mathrm{~s}^{-1}\right)}{\text { OOMK }}$ \\
\hline $\begin{array}{l}\text { casp-3 } \\
\text { casp-8 } \\
\text { casp-9 }\end{array}$ & $\begin{array}{l}0.013 \\
0.032 \\
0.079\end{array}$ & $\begin{array}{r}92000 \\
220 \\
410\end{array}$ & $\begin{array}{c}0.14 \\
150 \\
190\end{array}$ \\
\hline \multicolumn{4}{|c|}{$\begin{array}{l}\text { Ac-LETD-AOMK } \\
K_{i(\mathrm{nM})} k_{\text {inact }} / K_{i}\left(\mathrm{mM}^{-1} \mathrm{~s}^{-1}\right)\end{array}$} \\
\hline $\begin{array}{l}\text { casp-3 } \\
\text { casp-8 } \\
\text { casp-9 }\end{array}$ & $\begin{array}{l}0.0059 \\
0.14 \\
0.014\end{array}$ & $\begin{array}{r}12 \\
160 \\
11\end{array}$ & $\begin{array}{r}490 \\
880 \\
1300\end{array}$ \\
\hline
\end{tabular}

Figure 3. Calculated rate constants for reactions between CV8/9AOMK or Ac-LETD-AOMK and caspases-3, -8, or -9.

plotting progress curves of substrate turnover with respect to time in the presence and absence of inhibitor and deriving $k_{\mathrm{obs}}$ values for each reaction (Supplementary Figures S3-S5). ${ }^{28}$ Our initial efforts using CV8/9-KE were unsuccessful due to the low potency of this inhibitor against caspase- 3 even at high micromolar concentrations (data not shown). CV8/9-AOMK had no inhibitory activity against caspase-3 below $100 \mu \mathrm{M}$ without initial preincubation before substrate addition and was restricted to a limited window of concentrations for kinetic assessment (Supplementary Figures S3-S5). Moreover, the inability of CV8/9-AOMK to inhibit caspases-6 and -7 at low micromolar concentrations precluded their inclusion in this study.

The $k_{\text {inact }} / K_{i}$ ratio was then calculated and used to assess the ability of CV8/9-AOMK to selectively inhibit specific caspase isoforms (Figure 3). CV8/9-AOMK has similar $k_{\text {inact }}$ constants against caspases-3, -8 , and -9 , but $>100$-fold lower $K_{i}$ values for the initiator caspases- 8 and -9 versus the executioner caspase- 3 (Figure 3). These results indicate that discrimination against caspase- 3 is attributable to the CV8/9-AOMK recognition and reversible binding event with active site residues that results in the E-I reversible complex rather than covalent attachment to the catalytic cysteine and EI formation (Figure 3). ${ }^{28}$ Notably, $k_{\text {inact }} / K_{i}$ values were $>1000$-fold higher for CV8/9-AOMK against caspases- 8 and -9 with only a 3 -fold difference among caspases-3, -8, and -9 for Ac-LETD-AOMK (Figure 3). The significant CV8/9-AOMK selectivity for caspases-8 and -9 is also accompanied by a marked decrease in potency and coincides with our observations for the $\mathrm{IC}_{50}$ studies (Figures 2 and 3 ).

We determined the X-ray crystal structures of caspases- 3 and -8 in complex with CV8/9-AOMK to $1.63 \AA$ (green) and 1.73 $\AA$ (magenta) resolution, respectively, to shed light on differences in peptide binding orientations that promote selectivity (Figure 4, Supplementary Table S1). Superposition of the protein active sites uncovered significant deviations in the side-chain conformation of the CV8/9 P2 hydroxyproline (Hyp) between the two caspase structures. Hyp is forced into an unfavorable endo conformation when CV8/9 is bound to the caspase- 3 active site, and this results in a $142^{\circ}$ dihedral angle between the $\mathrm{N}-\mathrm{C} \delta-\mathrm{C} \gamma-\mathrm{O}$ atoms of the $\mathrm{P} 2$ residue (Figure 4). ${ }^{29}$ In contrast, Hyp is in the favorable exo conformation in the caspase- 8 structure as evidenced by an $86^{\circ}$ dihedral angle between the $\mathrm{N}-\mathrm{C} \delta-\mathrm{C} \gamma-\mathrm{O}$ atoms (Figure 4). ${ }^{29}$ The preferred CV8/9 P2 exo conformation is not achievable in caspase-3, as steric clashes would result between the Hyp hydroxyl moiety and active site residues Y204 and W206 (Figure 4). As such, the P2 Hyp is required to adopt the high-energy endo conformation in order to bind caspase-3, and these structural perturbations correlate well with the significantly increased $K_{i}$ values of reversible binding (Figure 3). Importantly, these large hydrophobic caspase- 3 residues are substituted with V410 and Y412 in caspase-8 that result in a larger active site pocket to accommodate P2 side chains despite Hyp adopting an exo conformation that directly points the five-membered ring and hydroxyl group into caspase-8 (Figure 4). The resulting N$\mathrm{C} \delta-\mathrm{C} \gamma-\mathrm{O}$ torsional strain for Hyp was determined using AMBER 14 molecular mechanics potential parameters to be 1.9 $\mathrm{kcal} / \mathrm{mol}$ greater for caspase-3 relative to caspase- 8 (Figure 4). ${ }^{29}$

AMBER 14 parameters were then assigned to each bond angle around the Hyp five-membered ring in order to identify angle strain from the exo versus endo conformations (Supplementary Figure S6). ${ }^{30}$ The $\mathrm{N}-\mathrm{C} \alpha-\mathrm{C} \beta$ bond angle had the largest discrepancy between caspases-3 $\left(96.6^{\circ}\right)$ and -8 $\left(102^{\circ}\right)$ compared to all other atoms, resulting in an energy difference of $2.8 \mathrm{kcal} / \mathrm{mol}$ for this forced position (Figure 4). The remaining bond angles had similar strain energies, providing a total bond angle strain difference of $2.5 \mathrm{kcal} / \mathrm{mol}$ between the Hyp side chain of $\mathrm{CV} 8 / 9$ that is bound to caspase- 


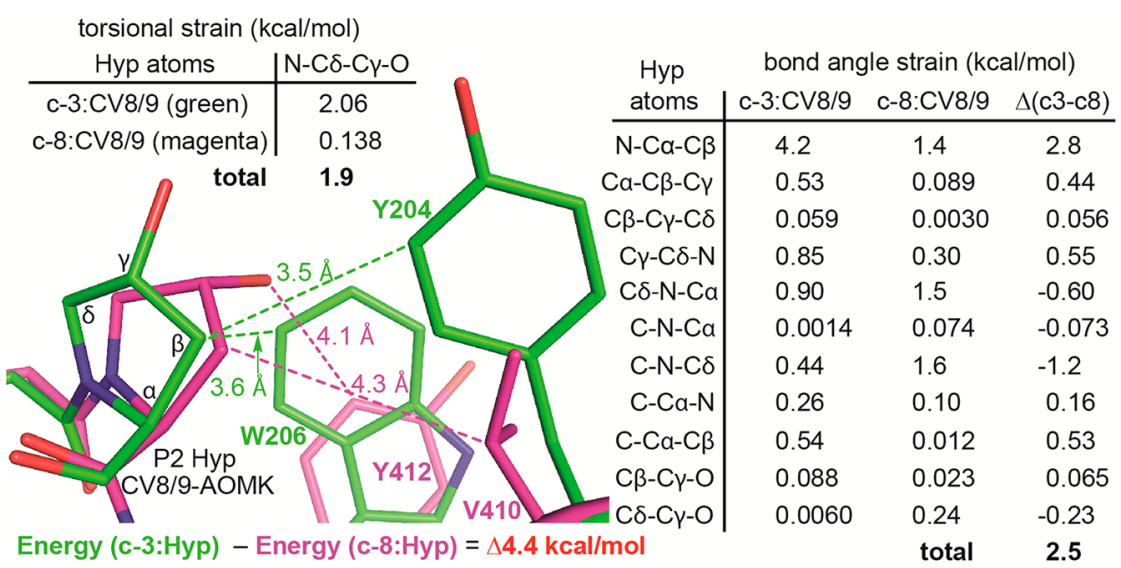

Figure 4. Conformational strain in the hydroxyproline (Hyp) side chain of CV8/9-AOMK observed in X-ray cocrystal structures in the active sites of caspases-3 (green, PDB ID: P4S0) and -8 (magenta, PDB ID: P4S1). Nitrogen and oxygen atoms are colored in blue and red, respectively.

3 versus -8 (Figure 4). Intriguingly, the Hyp residue alone was calculated to contribute a total energy difference of $4.4 \mathrm{kcal} /$ mol more for $\mathrm{CV} 8 / 9$ bound to caspase-3 in comparison to caspase-8 (Figure 4).

The importance of particular caspases for essential biological phenomena is typically studied using chemical probes and inhibitors that are based on canonical peptide recognition sequences that indiscriminately bind to multiple isoforms. These side effects significantly limit the resolution and convolute our understanding of how specific caspases function during human health and disease. In order to solve this problem, we devised an approach using unnatural amino acid libraries to develop a new class of chemical tools that are able to recognize only specific caspase isoforms. Through this study, we discover and characterize CV8/9-AOMK, an inhibitor of initiator caspases- 8 and -9 that discriminates against the executioner caspases-3, -6 , and -7 . Future work will advance our CV8/9 series compounds as chemical probes; however, initial attempts to modify the $\mathrm{N}$-terminus with fluorophores and biotin have resulted in a reduction in inhibitor selectivity (Supplementary Figure S7). This study provides a proof-ofconcept foundation for incorporating noncanonical amino acids in to discovery efforts for selective inhibitors and probes of many different caspase isoforms and other proteases.

\section{METHODS}

For methods see Supporting Information.

\section{ASSOCIATED CONTENT}

\section{S Supporting Information}

Supplementary figures and table. This material is available free of charge via the Internet at http://pubs.acs.org.

\section{AUTHOR INFORMATION}

\section{Corresponding Author}

*E-mail: wolan@scripps.edu.

\section{Notes}

The authors declare no competing financial interest.

\section{ACKNOWLEDGMENTS}

We thank Prof. H. Rosen for instrumentation. We also gratefully acknowledge financial support from The Scripps Research Institute and the National Science Foundation (predoctoral fellowship to C.J.V.).

\section{REFERENCES}

(1) Fuchs, Y., and Steller, H. (2011) Programmed cell death in animal development and disease. Cell 147, 742-758.

(2) Martin, S. J., Henry, C. M., and Cullen, S. P. (2012) A perspective on mammalian caspases as positive and negative regulators of inflammation. Mol. Cell 46, 387-397.

(3) Dorstyn, L., Puccini, J., Wilson, C. H., Shalini, S., Nicola, M., Moore, S., and Kumar, S. (2012) Caspase-2 deficiency promotes aberrant DNA-damage response and genetic instability. Cell Death Differ. 19, 1288-1298.

(4) Larsen, B. D., Rampalli, S., Burns, L. E., Brunette, S., Dilworth, F. J., and Megeney, L. A. (2010) Caspase 3/caspase activated DNase promote cell differentiation by inducing DNA strand breaks. Proc. Natl. Acad. Sci. U.S.A. 107, 4230-4235.

(5) Olson, N. E., Graves, J. D., Shu, G. L., Ryan, E. J., and Clark, E. A. (2003) Caspase activity is required for stimulated B lymphocytes to enter the cell cycle. J. Immunol. 170, 6065-6072.

(6) Williams, D. W., Kondo, S., Krzyzanowska, A., Hiromi, Y., and Truman, J. W. (2006) Local caspase activity directs engulfment of dendrites during pruning. Nat. Neurosci. 9, 1234-1236.

(7) Fujita, J., Crane, A. M., Souza, M. K., Dejosez, M., Kyba, M., Flavell, R. A., Thomson, J. A., and Zwaka, T. P. (2008) Caspase activity mediates the differentiation of embryonic stem cells. Cell Stem Cell 2, 595-601.

(8) Su, H., Bidére, N., Zheng, L., Cubre, A., Sakai, K., Dale, J., Salmena, L., Hakem, R., Straus, S., and Lenardo, M. (2005) Requirement for caspase- 8 in NF-kappaB activation by antigen receptor. Science 307, 1465-1468.

(9) Woo, M., Hakem, R., Furlonger, C., Hakem, A., Duncan, G. S., Sasaki, T., Bouchard, D., Lu, L., Wu, G. E., Paige, C. J., and Mark, T. W. (2003) Caspase-3 regulates cell cycle in B cells: a consequence of substrate specificity. Nat. Immunol. 4, 1016-1022.

(10) Rendl, M., Ban, J., Mrass, P., Mayer, C., Lengauer, B., Eckhart, L., Declerg, W., and Tschachler, E. (2002) Caspase-14 expression in epidermal keratinocytes is regulated by retinoids in a differentiationassociated manner. J. Invest. Dermatol. 119, 1150-1155.

(11) Pop, C., and Salvesen, G. S. (2009) Human caspases: activation, specificity, and regulation. J. Biol. Chem. 284, 21777-21781.

(12) Putt, K. S., Chen, G. W., Pearson, J. M., Sandhorst, J. S., Hoagland, M. S., Kwon, J.-T., Jin, H., Churchwell, M. I., Cho, M.-H., Doerge, D. R., Helferich, W. G., and Hergenrother, P. J. (2006) Smallmolecule activation of procaspase- 3 to caspase- 3 as a personalized anticancer strategy. Nat. Chem. Biol. 2, 543-550.

(13) Wolan, D. W., Zorn, J. A., Gray, D. C., and Wells, J. A. (2009) Small-molecule activators of a proenzyme. Science 326, 853-858.

(14) Vickers, C. J., González-Páez, G. E., Umotoy, J. C., CayananGarrett, C., Brown, S. J., and Wolan, D. W. (2013) Small-molecule procaspase activators identified using fluorescence polarization. ChemBioChem. 14, 1419-1422. 
(15) Leyva, M. J., Degiacomo, F., Kaltenbach, L. S., Holcomb, J., Zhang, N., Gafni, J., Park, H., Lo, D. C., Salvesen, G. S., Ellerby, L. M., and Ellman, J. A. (2010) Identification and evaluation of small molecule pan-caspase inhibitors in Huntington's disease models. Chem. Biol. 17, 1189-1200.

(16) Fulda, S., and Debatin, K.-M. (2006) Extrinsic versus intrinsic apoptosis pathways in anticancer chemotherapy. Oncogene 25, 47984811.

(17) Communal, C., Sumandea, M., de Tombe, P., Narula, J., Solaro, R. J., and Hajjar, R. J. (2002) Functional consequences of caspase activation in cardiac myocytes. Proc. Natl. Acad. Sci. U.S.A. 99, 62526256.

(18) Hotchkiss, R. S., and Nicholson, D. W. (2006) Apoptosis and caspases regulate death and inflammation in sepsis. Nat. Rev. Immunol. $6,813-822$

(19) Degterev, A., Boyce, M., and Yuan, J. (2003) A decade of caspases. Oncogene 22, 8543-8567.

(20) Mahrus, S., Trinidad, J. C., Barkan, D. T., Sali, A., Burlingame, A. L., and Wells, J. A. (2008) Global sequencing of proteolytic cleavage sites in apoptosis by specific labeling of protein N-termini. Cell 134, 866-876.

(21) Gray, D. C., Mahrus, S., and Wells, J. A. (2010) Activation of specific apoptotic caspases with an engineered small-moleculeactivated protease. Cell 142, 637-646.

(22) Vickers, C. J., González-Páez, G. E., and Wolan, D. W. (2013) Selective detection of caspase- 3 versus caspase-7 using activity-based probes with key unnatural amino acids. ACS Chem. Biol. 8, 15581566.

(23) Vickers, C. J., González-Páez, G. E., and Wolan, D. W. (2013) Selective detection and inhibition of active caspase-3 in cells with optimized peptides. J. Am. Chem. Soc. 135, 12869-12876.

(24) Han, Y., Giroux, A., Colucci, J., Bayly, C. I., Mckay, D. J., Roy, S., Xanthoudakis, S., Vaillancourt, J., Rasper, D. M., Tam, J., Tawa, P., Nicholson, D. W., and Zamboni, R. J. (2005) Novel pyrazinone monoamides as potent and reversible caspase-3 inhibitors. Bioorg. Med. Chem. Lett. 15, 1173-1180.

(25) Thornberry, N. A., Rano, T. A., Peterson, E. P., Rasper, D. M., Timkey, T., Garcia-Calvo, M., Houtzager, V. M., Nordstrom, P. A., Roy, S., Vaillancourt, J. P., Chapman, K. T., and Nicholson, D. W. (1997) A combinatorial approach defines specificities of members of the caspase family and granzyme B. J. Biol. Chem. 272, 17907-17911.

(26) Berger, A. B., Sexton, K. B., and Bogyo, M. (2006) Commonly used caspase inhibitors designed based on substrate specificity profiles lack selectivity. Cell Res. 16, 961-963.

(27) Berger, A. B., Witte, M. D., Denault, J.-B., Sadaghiani, A. M., Sexton, K. M. B., Salvesen, G. S., and Bogyo, M. (2006) Identification of early intermediates of caspase activation using selective inhibitors and activity-based Probes. Mol. Cell 23, 509-521.

(28) Wang, Z., Watt, W., Brooks, N. A., Harris, M. S., Urban, J., Boatman, D., McMillan, M., Kahn, M., Heinrikson, R. L., Finzel, B. C., Wittwer, A. J., Blinn, J., Kamtekar, S., and Tomasselli, A. G. (2010) Kinetic and structural characterization caspase- 3 and caspase-8 inhibition by a novel class of irreversible inhibitors. Biochim. Biophys. Acta 1804, 1817-1831.

(29) Park, S., Radmer, R. J., Klein, T. E., and Pande, V. S. (2005) A new set of molecular mechanics parameters for hydroxyproline and its use in molecular dynamics simulations of collagen-like peptides. J. Comput. Chem. 26, 1612-1616.

(30) Ho, B. K., Coutsias, E. A., Seok, C., and Dill, K. A. (2005) The flexibility in the proline ring couples to the protein backbone. Protein Sci. 14, 1011-1018. 